5 January 2016

\title{
Benchmark and combined velocity-space tomography of fast-ion D-alpha spectroscopy and collective Thomson scattering measurements
}

\author{
A.S. Jacobsen ${ }^{1}$, M. Salewski ${ }^{1}$, B. Geiger ${ }^{2}$, S.B. Korsholm ${ }^{1}$, \\ F. Leipold ${ }^{1}$, S.K. Nielsen ${ }^{1}$, J. Rasmussen ${ }^{1}$, M. Stejner ${ }^{1}$, \\ M. Weiland ${ }^{2}$ and the ASDEX Upgrade team ${ }^{2}$ \\ 1 Technical University of Denmark, Department of Physics, DK-2800 Kgs. Lyngby, \\ Denmark \\ ${ }^{2}$ Max Planck Institute for Plasma Physics, D-85748 Garching, Germany \\ E-mail: ajsen@fysik.dtu.dk
}

\begin{abstract}
We demonstrate the combination of fast-ion D-alpha spectroscopy (FIDA) and collective Thomson scattering (CTS) measurements to determine a common best estimate of the fast-ion velocity distribution function by velocity-space tomography. We further demonstrate a benchmark of FIDA tomography and CTS measurements without using a numerical simulation as common reference. Combined velocity-space tomographies from FIDA and CTS measurements confirm that sawtooth crashes reduce the fast-ion phase-space densities in the plasma center and affect ions with pitches close to one more strongly than those with pitches close to zero.
\end{abstract}

\section{Introduction}

ASDEX Upgrade is equipped with several fast-ion diagnostics [1]. Collective Thomson scattering (CTS) [2-5] and fast-ion $\mathrm{D}_{\alpha}$ (FIDA) spectroscopy [6-9] are sensitive to the fast-ion velocity distribution function in small measurement volumes. Neutron emission spectroscopy (NES) [10], neutral particle analyzers [11] and gamma-ray spectroscopy (GRS) [12] are sensitive to the fast-ion velocity distribution function along their linesof-sight. Fast-ion loss detectors are sensitive to ions ejected from the plasma [13].

FIDA measurements can be interpreted using velocity-space tomography [14-17]. With this method one determines the velocity distribution function $F$ from the measurements $S$ by solving the discrete inverse problem $S=W F$ where $W$ is a matrix composed of so-called weight functions $[18,19]$. These reveal the velocityspace sensitivity of the measurements. The method has recently been demonstrated experimentally [7] and has since been applied to study sawtooth crashes $[9,17,20]$. Theoretical expectations [21,22] and previous experiments on TEXTOR using CTS [23] and on DIII-D and ASDEX Upgrade using FIDA $[8,24]$ indicated that sawtooth crashes eject fast ions with pitches close to one more strongly than those with pitches close to 


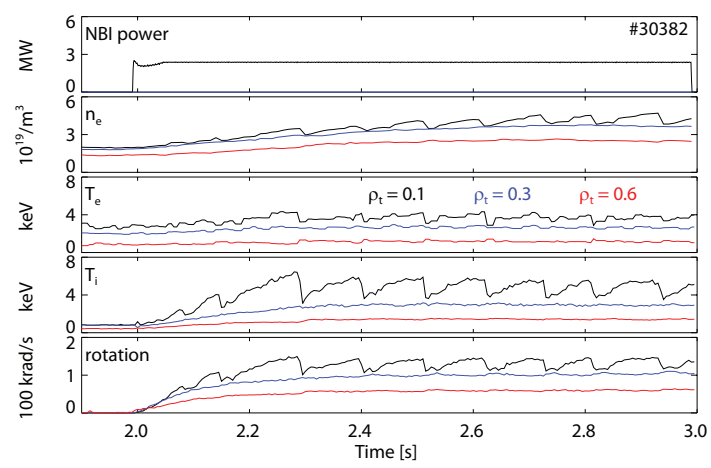

Figure 1. Overview of discharge \#30382 showing the neutral beam injection (NBI) power, electron density, electron temperature, ion temperature and plasma rotation.

zero from the plasma center. FIDA tomographies of 2D fast-ion velocity distribution functions allow the calculation of the sawtooth-induced redistribution as a function of energy and pitch of the fast ions, confirming the pitch dependence of sawtooth crashes $[9,17,20]$. Simultaneous FIDA tomographies inside and outside the sawtooth inversion radius showed that this decrease of the phase-space densities inside the inversion radius is accompanied by a corresponding increase outside [20]. A reduction of the fast-ion density above a threshold energy of $10 \mathrm{keV}$ in the plasma center could further be calculated [9].

Here we experimentally demonstrate firstly a method to benchmark and secondly a method to combine different fast-ion diagnostics using simultaneous FIDA and CTS measurements at ASDEX Upgrade. Consistency checks across fast-ion diagnostics are difficult as the diagnostics observe different regions in position space as well as in velocity space. Therefore, consistency has usually been assessed by comparing the measurements with synthetic measurements based on TRANSP/NUBEAM simulations as common reference $[4,18,25]$. Velocity-space tomography allows us to benchmark CTS and FIDA measurements without simulations. Further, we experimentally demonstrate a combination of different fast-ion diagnostics using velocity-space tomography of simultaneous FIDA and CTS measurements [15].

\section{Benchmark of FIDA tomography and CTS}

In this section we calculate synthetic CTS measurements implied by a FIDA tomography from simultaneously acquired spectra from four views and compare them with actual CTS measurements in discharge \#30382 [26]. The discharge was heated by the $60 \mathrm{keV}$ neutral beam injector Q3 at 2.5 MW. Figure 1 shows an overview of main plasma parameters revealing the presence of sawteeth as periodic variations in electron density, ion temperature and toroidal rotation. Figure 2 shows simultaneously acquired spectra from four FIDA views before and after the large sawtooth crash at $t=2.29 \mathrm{~s}$. The sawtooth crash leads to reductions in the measured FIDA intensities. The measurement volumes of the four FIDA views are at a normalized toroidal flux of $\rho_{t}=0.1$. The FIDA spectra depend not only on the fast-ion phase-space densities but also on nuisance 
parameters such as the electron density which themselves show sawtoothing behaviour (figure 1). FIDA tomographies account for any changes in nuisance parameters. The beam deposition profile does not change significantly during a sawtooth crash as the beam emission in the central measurement volume remains approximately constant [9]. We reject spectral ranges dominated by beam emission or impurity lines. The beam emission typically dominates the FIDA emission at moderate Doppler shifts. Here the strongest impurity transitions are Boron V $(n=10 \rightarrow 8)$, Oxygen II, Oxygen V and Helium II. We also reject spectral ranges in the spectra that are sensitive to ions below $10 \mathrm{keV}$ (thermal ions) or only to ions above $80 \mathrm{keV}$ as we do not attempt to reconstruct these regions. The rejected spectral ranges are shown in figure 2. As the NBI was not modulated, a standard subtraction of background noise and impurity lines in the FIDA spectra is not applicable. This decreases the usable wavelength ranges. The low density $\left(n_{e}=3 \times 10^{19} \mathrm{~m}^{-3}\right)$ makes up for this shortcoming due to the resulting high signal-to-noise ratio of the FIDA measurements.

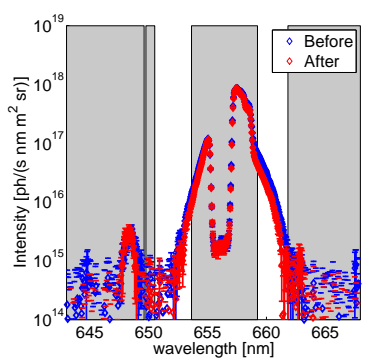

(a) $\phi=14^{\circ}$

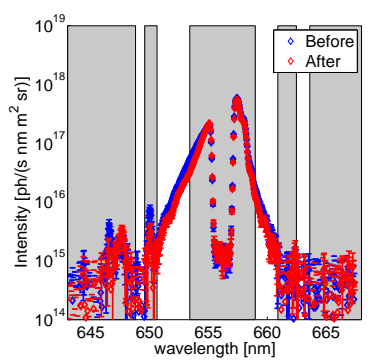

(b) $\phi=129^{\circ}$

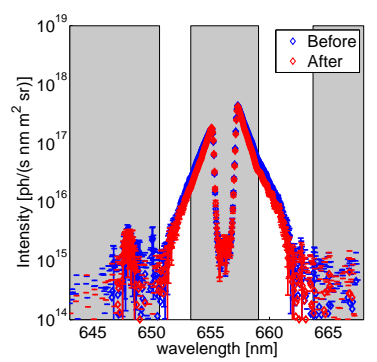

(c) $\phi=70^{\circ}$

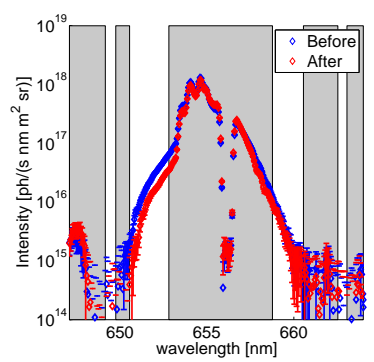

(d) $\phi=155^{\circ}$

Figure 2. Simultaneously measured FIDA spectra in four views before and after a sawtooth crash at $t=2.29 \mathrm{~s}$ in discharge \#30382. The shaded regions are rejected. $\phi$ is the angle between the line-of-sight and the magnetic field.

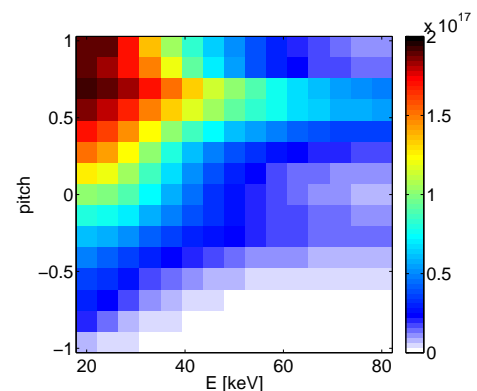

(a) Before

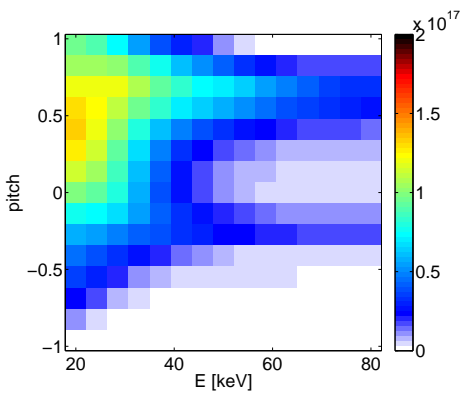

(b) After

Figure 3. FIDA tomographies [ions $/ \mathrm{m}^{3} / \mathrm{keV}$ ] before and after a sawtooth crash at $t=2.29 \mathrm{~s}$ in discharge \#30382 based on the spectra from figure 2.

Figure 3 shows FIDA tomographies before and after the sawtooth crash as calculated from the FIDA spectra in figure 2 using the minimum Fisher information regularization method on a $15 \times 15$ grid [17]. The FIDA tomographies allow the 
computation of synthetic CTS measurements, i.e. 1D velocity-distribution functions $g(u)$ which are projections of the full distribution function onto the plasma fluctuation wave vector [27]. These synthetic CTS measurements are shown together with the actual CTS measurements before and after the sawtooth crash in figure 4 . The synthetic and actual CTS measurements include the injected fast ions as well as the fast ions found in the tails of the thermal ion distribution. The synthetic CTS measurements match the actual CTS measurements well within the uncertainties. The synthetic CTS measurements tend to lie marginally above the actual CTS measurements which could be explained by calibration uncertainty or by the presence of impurity light that is misinterpreted as FIDA light. Another possible explanation is that the FIDA measurement volume is slightly on the low field side whereas the CTS measurement volume is slightly on the high field side. Hence trapped particles might not reach the CTS measurement volume. However, it should be noted that the measurements are central with $\rho_{t}<0.1$.

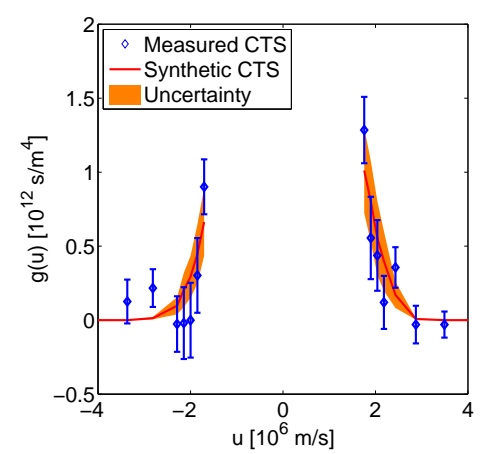

(a) Before

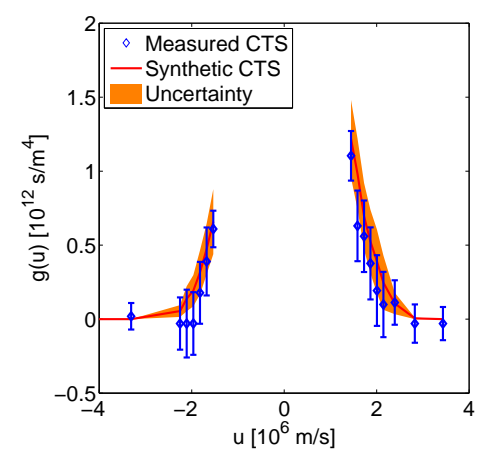

(b) After

Figure 4. Comparison of real CTS measurements and synthetic CTS measurements implied by the FIDA tomographies in figure 3. The uncertainties include measurement noise, uncertainties of nuisance parameters and, for the synthetic CTS measurements based on FIDA tomography, the bias due to the regularization [17].

\section{Combination of CTS and FIDA in velocity-space tomography}

Velocity-space tomography allows us to combine measurements from different fast-ion diagnostics to calculate a common best estimate of the fast-ion distribution function [15]. Here we present the first experimental demonstration. For such a combination, the normalization of the measurements and of the weight function matrix with the estimated uncertainties becomes crucial [15]. These uncertainties need to be defined consistently for the different diagnostics. CTS uncertainties account for uncertainties in the measured spectral power densities as well as in the nuisance parameters used in the fit of $g(u)[2,4,5]$. Uncertainties in FIDA intensities can be calculated based on the photon noise. However, the uncertainties in the nuisance parameters are often not accounted for. Here we need to account for these uncertainties to treat FIDA and CTS 
on an equal footing. They enter the problem as uncertainty in the weight functions. The FIDA weight functions depend on the electron temperature and density, the ion temperature and drift velocity and the effective charge. Here we estimate the uncertainty due to these nuisance parameters as demonstrated in reference [17].

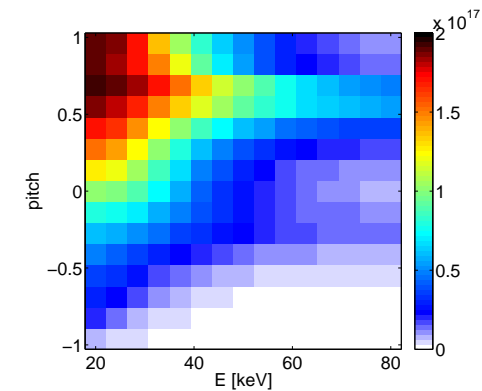

(a) Before

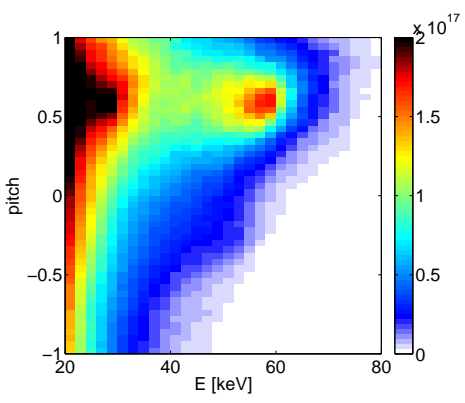

(d) TRANSP before

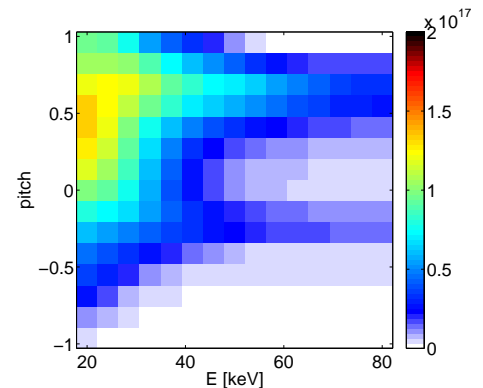

(b) After

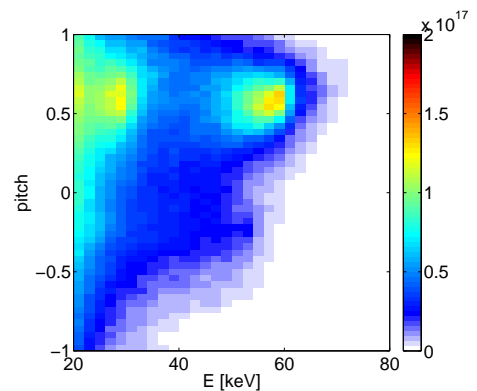

(e) TRANSP after

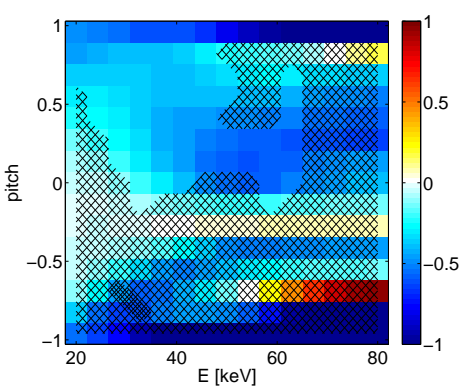

(c) $\Delta F_{\text {rel }}$

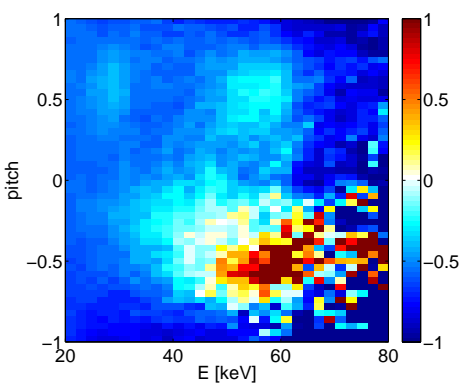

(f) TRANSP $\Delta F_{\text {rel }}$

Figure 5. Velocity-space tomographies [ions $/ \mathrm{m}^{3} / \mathrm{keV}$ ] from combined FIDA and CTS measurements before and after the sawtooth crash at $t=2.29 \mathrm{~s}$ (as in figure 3 ) as well as the relative change. The lower row shows corresponding TRANSP simulations. In the hatched area in (c) the uncertainties on the relative change (see figure 6) are larger than the relative change.

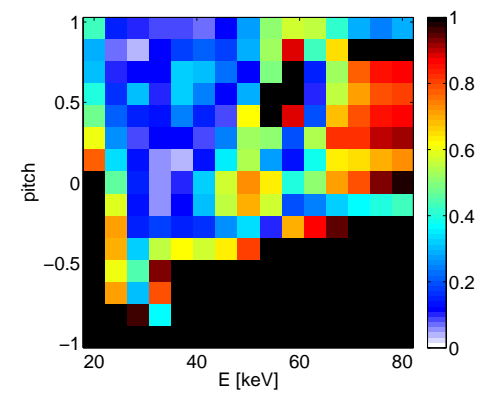

Figure 6. Uncertainty of the relative crash size based on velocity-space tomographies before and after the sawtooth crash. The uncertainties acount for photon noise, nuisance parameters and the bias due to the regularization [17]. Regions with large uncertainties compared with the crash size are shown as hatched regions in figure 5 .

Figure 5 shows tomographies based on combined FIDA and CTS measurements before and after the sawtooth crash together with a corresponding TRANSP simulation. 
We neglect the small difference in the spatial locations of the measurement volumes as all volumes are central with $\rho_{t}<0.1$. We further show the measured and simulated relative crash sizes $\Delta F_{\text {rel }}=\left(F_{\text {after }}-F_{\text {before }}\right) / F_{\text {before }}$ resolved in velocity space. The fast-ion densities are strongly reduced due to the crash. This reduction is stronger for pitches close to one than for pitches close to zero for energies below about $40 \mathrm{keV}$. The tomographies confirm previous results based on FIDA tomographies from five views which showed, however, a stronger pitch dependence $[9,17,20]$. The energy-pitch dependence of the sawtooth crash in the TRANSP simulation and the measurement is quite different. The relative crash size can be calculated throughout velocity space since the fast-ion densities in the tomography as well as in the TRANSP simulation throughout velocity space are larger than zero. However, the values in the hatched areas have large uncertainties. Figure 6 shows the estimated uncertainty of the relative change based on uncertainties in the tomographies and standard error propagation. The uncertainties represent photon noise, uncertainty due to nuisance parameters and the bias due to the regularization [17]. The relative change is reliable for positive pitch and energies below the injection energy.

A comparison of figure 5(a)-(b) and figure 3 shows that the addition of the CTS measurements does not change the tomographies appreciably. The inferred relative crash sizes as well as their uncertainties change marginally by about $\pm 1 \%$. Slight increases in uncertainty likely result from the systematic uncertainties discussed in section 2 . The small effect of CTS is expected in this discharge for three reasons. First, the FIDA measurements consist of 487 usable data points while the CTS measurements consist of 18. In the future the number of CTS measurements could be significantly increased by using high frequency resolution CTS measurements [28] and two simultaneous CTS views. Second, the uncertainty in FIDA measurements is about $5 \%$ of the total signal whereas the uncertainty of CTS measurements is about $20 \%$. Third, the reasonable matches between the FIDA-implied synthetic CTS measurements and the actual CTS measurements suggest that the CTS measurements agree with the FIDA tomography and thus should not change it much.

\section{Discussion and Conclusions}

We have experimentally demonstrated that FIDA measurements from four views and CTS measurements from one view can be combined to give a common best estimate of the 2D fast-ion distribution function using velocity-space tomography. We further benchmarked CTS measurements against synthetic measurements implied by FIDA tomography without the need for fast-ion simulations as common reference. Our methods are directly applicable to LHD which is equipped with CTS $[29,30]$ and two FIDA views [31]. The availability of six FIDA views and two CTS views at ASDEX Upgrade will further improve the inversion for studies of for example Alfvén eigenmodes or the effect of ion cyclotron resonance heating. As our approach relies on weight functions for FIDA $[18,19]$ and CTS [32], it will, together with recently derived NES 
weight functions [33] and GRS weight functions [34], allow us to combine GRS and NES measurements at JET [35] in velocity-space tomography of fusion alphas in the upcoming JET D-T campaign [36]. Lastly, ITER will be equipped with GRS, NES and CTS diagnostics. A helium variant of FIDA could provide additional measurements. Thus, the methods of benchmarking and combination of fast-ion diagnostics experimentally demonstrated here will apply to ITER. We demonstrated our approach by benchmarking and combining FIDA and CTS measurements before and after a sawtooth crash at ASDEX Upgrade. Previous results of the crash size and the velocity-space resolved ejection of fast ions from the center based on FIDA tomography could be confirmed $[8,17,20]$.

\section{Acknowledgments}

This work has been carried out within the framework of the EUROfusion Consortium and has received funding from the Euratom research and training programme 20142018 under grant agreement No 633053. The views and opinions expressed herein do not necessarily reflect those of the European Commission.

\section{References}

[1] Stroth U et al 2013 Nucl. Fusion 53104003

[2] Salewski M et al 2010 Nucl. Fusion 50035012

[3] Furtula V et al 2012 Rev. Sci. Instrum. 83013507

[4] Rasmussen J et al 2015 Plasma Phys. Control. Fusion 57075014

[5] Nielsen S K et al 2015 Plasma Phys. Control. Fusion 57035009

[6] Geiger B et al 2011 Plasma Phys. Control. Fusion 53065010

[7] Salewski M et al 2014 Nucl. Fusion 54023005

[8] Geiger B et al 2015 Plasma Phys. Control. Fusion 57014018

[9] Geiger B et al 2015 Nucl. Fusion 83083001

[10] Tardini G et al 2012 Journal of Instrumentation 7 C03004-C03004

[11] Schneider P A et al 2015 Rev. Sci. Instrum. 86073508

[12] Nocente M et al 2012 Nucl. Fusion 52094021

[13] García-Muñoz M et al 2007 Nucl. Fusion 47 L10-L15

[14] Salewski M et al 2012 Nucl. Fusion 52103008

[15] Salewski M et al 2013 Nucl. Fusion 53063019

[16] Salewski M et al 2015 Plasma Phys. Control. Fusion 57014021

[17] Jacobsen A S et al Plasma Phys. Control. Fusion Inversion methods for fast-ion velocity-space tomography in fusion plasmas

[18] Heidbrink W W et al 2007 Plasma Phys. Control. Fusion 49 1457-1475

[19] Salewski M et al 2014 Plasma Phys. Control. Fusion 56105005

[20] Weiland M et al Plasma Phys. Control. Fusion Enhancement of the FIDA diagnostic at ASDEX Upgrade for velocity space tomography

[21] Kolesnichenko Y et al 2000 Nucl. Fusion 40 1325-1341

[22] Jaulmes J et al $201514^{\text {th }}$ IAEA Technical Meeting on Energetic Particles

[23] Nielsen S K et al 2011 Nucl. Fusion 51063014

[24] Muscatello C M et al 2012 Plasma Phys. Control. Fusion 54025006

[25] Heidbrink W W et al 2014 Plasma Phys. Control. Fusion 56095030 
[26] Rasmussen J et al $201514^{\text {th }}$ IAEA Technical Meeting on Energetic Particles

[27] Moseev D et al 2011 Plasma Phys. Control. Fusion 53105004

[28] Stejner M et al 2015 Plasma Phys. Control. Fusion 57062001

[29] Kubo S et al 2010 Rev. Sci. Instrum. 81 10D535

[30] Nishiura M et al 2014 Nucl. Fusion 54023006

[31] Ito T et al 2010 Rev. Sci. Instrum. 81 10D327

[32] Salewski M et al 2011 Nucl. Fusion 51083014

[33] Jacobsen A S et al 2015 Nucl. Fusion 55053013

[34] Salewski M et al 2015 Nucl. Fusion 55093029

[35] Eriksson J et al 2015 Nucl. Fusion 55123026

[36] Romanelli F 2015 Nucl. Fusion 55104001 